\title{
基于积极心理学的初中生心理健康教育校本课程的实践与思考
}

禇容正

绍兴市锡麟中学

DOI:10.32629/er.v2i10.2091

[摘 要] 为了有效的保障初中生的心理健康, 本文将对开发初中心理健康校本课程的背景、课程的内容以及核心理念、课程的定位, 实施与评 估课程等方面的问题进行实践,对实施校本课程的成果进行总结,并且对其中存在的不足进行分析,争取能够不断完善校本课程。

[关键词] 积极心理学; 心理健康教育; 校本课程

积极心理学是心理学的分属, 经过心理学的引导, 可以使人们对健康、 勇气、关爱进行深入的研究。所以在积极心理学的背景下, 对初中生进行 心理健康教育, 能够使初中生的心理健康得到有效的保障。

\section{1 开发课程的背景}

1. 1 积极心理学的发展

作为近年来新兴心理思潮的积极心理学, 为传统的心理学提供了新的 活力, 在它的发展过程中, 吸引了大量心理学者的兴趣和关注, 并且取得了 较好的研究成果。我国的积极心理学还处于研究的阶段, 在国内积极心理 学并没有得到广泛的应用以及研究。现阶段, 在探索和实践积极心理学方 面, 特别是在初中教育阶段的应用相对薄弱, 所以, 在积极心理学的背景下, 开发初中生心理健康教育校本课程具有非常重要的意义 ${ }^{[1]}$ 。

1. 2 学校心理健康教育的现状

第一, 在学校传统的心理健康教育体系中往往是以问题为导向, 就是 对青少年的问题过于关注, 而对培养学生积极心理和自身优势有所忽略, 这是一种比较被动与消极的心理健康教育模式, 应该对学生的自我优势进 行重视, 使学生的自身潜能够得到最大程度的发挥, 对学生积极的心理品 质进行培养, 使学生能够以积极的心态面对学习生活中的难题。

第二, 很多学校不够重视心理课程, 没有对心理课程进行足量的开设。 学校想要使教育目标得到实现, 进行课堂教学是其中最有效的载体 ${ }^{[2]}$ 。现 阶段, 仍然还有中学不开设对学生积极心理品质进行培养的课程, 有的学 校由于环境较差缺少专门的心理教学器材以及心理教师。这些学校设置的
课程无法使中学生的各种心理诉求得到有效的满足。相对落后的心理课程, 对发展学生心理健康教育起到了一定程度的阻碍作用。

第三, 城市化进程的影响。随着快速发展的城市化进程, 使很多城乡 结合部出现, 在城乡结合部中具有非常频繁的人口流动, 复杂的生活环 境, 在这个区域内生活的初中生普遍存在行为、心理等方面的问题, 会出 现人际关系敏感、厌学、离家出走、反抗心理等表现, 心理教育工作者 应该对这些初中生的内心世界进行特别关注, 对它们积极心理品质进行 培养, 使它们能够收获更多的积极情绪和积极体验, 为他们幸福、健康的 成长提供保障。

1.3 在心理健康教育中积极心理学的作用

科学主义以及人文主义是积极心理学的基本内容, 积极心理学填补了 传统心理学教育中的缺陷, 相对于消极心理学, 积极心理学对人的心理品 质更加注重, 能够为人们解决思想问题以及心理问题提供有效的帮助, 帮 助人完成良好思想品质以及行为作风的塑造 ${ }^{[3]}$ 。应用积极心理学, 能够使 心理健康教育变得更加完善。

\section{2 课程的内容以及核心理念设置}

2.1设置课程的内容

首先, 需要对课程目标进行明确, 帮助学生完成良好自我观的建立, 使 学生能够对自身的情绪进行调控, 使学生能够以积极的态度看待问题、解 决问题, 使学生完成积极问题处理模式的建立, 使学生在生活中出现难题 时能够以积极乐观的态度面对, 对学生积极的心理品质进行培养, 使学生

的文学思维构件具有非同寻凡的意义。经常性地阅读文学大师们的作品, 可 以在一定程度上使得自身的文学修养得到不断地提高, 可以说, 对高中生而 言, 阅读文学大师的作品可以增强学生们对语文的运用能力, 其对培养学生 实用性阅读能力起到了很大的帮助性作用。文学大师作品可以称得上被世 界各国学者公认的经典之作, 譬如: 中国的四大古典名著、英国莎士比亚的 戏剧、中国现代时期鲁迅先生的名世之作等等, 伴随着时代的变迁, 这些经 典的作品名著可以说在阅读的时候需要花费很长的一段时间, 在这种情况 下我们要养成制定阅读计划的好习惯, 有安排的进行名著的阅读, 保证每一 部著作阅读的质量, 在阅读完后写读后感, 这样不仅可以提高自身的阅读能 力, 并且在文字语言的理解能力方面也会得到非常明显地提高。

\section{4 把握各类文章体裁的阅读能力}

作为一名优秀的高中生实用性阅读能力的培养要尽可能地阅读各类 体裁的文章, 通过阅读来感受不同形式下文字带来的不一样的感悟。大量 的阅读训练在一定程度上可以提高高中生对文字语言的敏锐性, 加强自身 对文章本质内涵的理解与认知。语文阅读可以说是非常开心的事情, 帮助 高中生把握好各类文章体裁的阅读方向, 不断提高他们在语文实用性阅读 方面的能力, 不仅能够培养高中生认真阅读的习惯, 同时能够养成一边读

书一边思考的能力, 可以对主要问题进行重点的思考, 从而提高阅读效率, 得到更多地收获。

\section{5 结束语}

从高中生的角度来讲, 实用性阅读是非常重要的一项能力, 可以说关 乎着未来高中生的个人发展方向, 所以说, 对高中生语文实用性阅读能力 培养进行探究具有非常重要的现实意义。日常中对高中生语文实用性阅读 能力的培养方式可以说多种多样, 但其中的核心方向是侧重对高中生阅读 兴趣、阅读习惯的培养, 这样才能够让学生们明白广泛阅读的意义, 明白增 强自身实用性阅读能力的重要价值。

[参考文献]

[1]肖笛.浅谈高中生语文实用性阅读能力培养[J].科学大众(科学教 育),2019,(09):4.

[2]朱一唯. 基于交际能力培养的高中语文教学研究[J]. 读与写(教育教 学刊),2019,16(08):77-78.

[3]宋宏平. 浅议高中生语文阅读能力的培养 [J]. 甘肃教 育,2019,(07):59.

[4]朱戌洋.高中生语文阅读学习探究[J].课程教育研究,2019,(12):78. 
能够很好的适应社会, 能够积极的追求自身的理想。

另外, 应该对课程的内容进行确定, 一方面培养学生的应变能力, 另一 方面是通过团体活动对学生积极的心理品质进行培养 ${ }^{[4]}$ 。培养学生的应变 能力包括以下内容: 良好自我观的建立、小组破冰活动、对个人力量进行 充分认识, 对自信心进行增强, 使学生能够保持冷静的心态, 对自己的支持 网络进行识别和使用, 东得换位思考、与他人保持和谐的关系; 培养积极 的心理品质包括: 创造力、领导力、心智、坚韧的毅力、勇敢、团队精神、 怜悯和宽容、被爱与爱的能力、自制能力、乐观和幽默、交流能力等。

2. 2 课程的核心理念

在对校本课程进行设置时, 应该结合积极心理学的理念和方法, 所以 与传统的教学模式相比, 应该对学生获得的积极情绪以及参与体验更加关 注, 通过对团体活动进行组织, 可以对学生积极的心理品质进行培养, 如勇 气、创造力等 ${ }^{[5]}$ 。开设课程的目的是对学生积极的心理品质进行培养, 所 以应该对学生的优势进行特别关注, 使学生的潜能得到充分发挥。

在实施校本课程的过程中, 应该对学生的个体差异进行充分考虑, 对 交流和教学过程进行关注, 对学生的收获以及心理体悟进行关注 ${ }^{[6]}$ 。心理 教师应该对教师的角色进行淡化, 以同伴的身份与学会进行良好互动, 使 学生的人格能够得到健康的发展。

\section{3 课程定位}

3. 1 对学生的应变能力以及积极的心理品质进行培养

以往人们对学生的问题过度关注, 学校也在使用比较消极的心理教育 模式。与传统的心理教育模式相比, 校本课程具有很大的不同, 需要对积极 心理健康教育的理念进行遵循, 对学生的积极优势更加关注, 通过积极的 对学生进行暗示, 使学生积极的情绪得到激发, 使他们能够正视并且放大 自身的优势, 能够对自身的情绪进行良好的调控, 促进问题积极处理模式 的树立, 使学生的人际关系更加和谐, 对学生的发展起到推动的作用 ${ }^{[7]}$ 。

3. 2师生共同进步

初中是人生中的一个重要的过度阶段, 使树立人生观、价值观、世界 观的重要时期, 也是心里走向成熟和构建自身人格的重要时期, 对于中学 教师来说, 也应该对自身的生命价值进行充分发挥。只有教师具有积极的 心理才能培养出相同的学生, 实现师生共同进步 ${ }^{[8]}$

\section{4 实施课程}

4. 1 对任课教师进行确定并且培训

选择具有较高综合心理素质且拥有学习心理学兴趣的教师作为任课 教师。并且需要组织这些认可教师进行专业的培训, 使这些教师能够满足 教学需求。

4. 2 对实施课程的方法进行确定

对学生进行校本课程的时间进行利用, 对心理健康教育校本课程进行 开展, 每节课程使用正常的课程时间。

4. 3对实施课程的方式进行确定

以一名任课教师教导十个学生的方式进行。活动场地可以在专门的心 理教室或者体育馆等, 需要保持授课空间的安静、独立。
4. 4对课程计划进行制定核对教学活动进行组织

每学期初, 应该对该学期实施校本课程的方案进行制定, 包括备课的 地点时间、师资安排、分析会议等。

4.5 合理的开发和使用课程资源

根据教学内容, 对配套的教学资源进行开发, 如, 活动方案、教案、活 动道具、活动视频等。使教学设计和课程资源实现多样化, 使教师和学生 的个性发展需求得到满足。同时应该对收集课后反馈进行重视, 使教师能 够及时的调整和修改课程。

\section{5 评估教学效果}

在评估教学效果方面, 心理健康教育与其他学科存在不同。由于受到 多方面因素的影响, 无法使用标准化测试对心理健康教育进行评估。随着 快速发展的积极心理学理念, 可以采取以下方法对教学效果进行评估。1. 教师记录和评价学生的课堂表现; 2. 对学生进行问卷调查; 3. 学生评价课 堂效果 ${ }^{[9]}$ 。通过分析, 在积极心理学的背景下进行初中生心理健康教育校 本课程, 能够取得非常好的效果, 能够对中学生积极的心理品质进行有效 的培养, 使中学生以积极的态度面对今后出现的问题, 有利于中学生以后 的发展。

\section{6 结束语}

综上所述, 开设基于积极心理学的初中生心理健康教育校本课程, 能 够对初中生不良的心理状态进行有效的调节, 使初中生以积极的态度看待 问题, 以积极的方法解决问题。所以, 相关的教育工作者可以从完善课程评 价体系、优化课程内容等方面进行改进, 进一步完善与加强校本课程体系, 争取取得更好的心理健康教育效果。

\section{[参考文献]}

[1]孙昊.学校心理健康教育校本课程开发的实践与探索[J].中小学心 理健康教育,2017,(12):26-28.

[2]何雪芬. 基于积极心理品质提升的心理类校本课程建设探究 [J]. 天 津电大学报,2019,23(01):67-69.

[3]鲍玮.积极心理学视角下小学心理校本课程创新实践 [J].中小学心 理健康教育,2018,378(31):79-82.

[4]袁春燕.积极心理学视野下进行心理健康教育校本课程开发的尝 试[J].广西教育,2015,(1):19-20.

[5]李厚仪. 基于积极心理学的心理健康教育在高校的应用——从理 论构建到实践探索[J].统计与管理,2017,(12):70-72.

[6]郑治国,刘建平.网络境遇下小学生性心理健康教育:基于积极心理 学的视野 [C].中部心理学高峰论坛,2012-8-1.

[7]王立高.基于积极心理学的高职心理健康教育课程与教学改革思 考[J].中国校外教育,2015,(3):145-146.

[8]朱其志,倪涵,涂冬侠.基于积极心理学的心理健康教育本土化发展 思考[J].中国教育技术装备,2017,(21):80-81.

[9]徐彩意, 孙小兵. “家校合作开展初中生心理健康教育”校本课程的 开发[J].教学月刊・中学版(语文教学),2014,(8):44-46. 